Revue

Revue de l'histoire des religions

del'histoire des religions

L'objet rituel. Concepts et méthodes croisés

\title{
Images, rites et magie aux marges des églises dans l'Occident médiéval
}

Images, rituals and magic on the fringes of churches in the medieval West

\section{Alessia Trivellone}

\section{OpenEdition \\ Journals}

Édition électronique

URL : http://journals.openedition.org/rhr/8333

DOI : $10.4000 /$ rhr.8333

ISSN : 2105-2573

\section{Éditeur}

Armand Colin

Édition imprimée

Date de publication : 1 décembre 2014

Pagination : 775-796

ISSN : 0035-1423

\section{Référence électronique}

Alessia Trivellone, «Images, rites et magie aux marges des églises dans l'Occident médiéval », Revue de l'histoire des religions [En ligne], 4 | 2014, mis en ligne le 01 décembre 2017, consulté le 10 décembre 2020. URL : http://journals.openedition.org/rhr/8333 ; DOI : https://doi.org/10.4000/rhr.8333 


\section{Images, rites et magie aux marges des églises dans l'Occident médiéval}

L'article analyse un ensemble d'images monumentales du Moyen Âge qui ont comme dénominateur commun d'être placées à l'extérieur d'édifices et de posséder des pouvoirs sans figurer la divinité. Si certaines sont le support d'incantations magiques, d'autres témoignent de rites, alors qu'un autre groupe représente des sujets qui leur confèrent des propriétés performatives. En dépit de ces différences, ces images fonctionnent selon des principes d'efficacité communs. Certains, comme la similitudo ou l'évocation des défunts, sont propres à la magie. Ces images, agissant sur l'espace et sur les hommes à des fins différentes, créent ainsi des "dispositifs ritualisants» aux marges de l'espace ecclésial.

\section{Images, rituals and magic on the fringes of churches in the medieval West}

The article analyzes a small group of performative images from the exterior monumental decoration of buildings in the Middle Ages that do not represent the divinity. Some of them were the object of magical incantations, others are simply the trace of rites and another group represents subjects that confer power on them. In spite of these differences, these images work according to common principles of efficiency. Some of them, such as the similitudo or evocation of the dead, are specific to the functioning of magic. These images, acting on space and on people with various purposes, create "ritualizing devices" in the margins of the ecclesial space. 
Au Moyen Âge, les images vivent et agissent: il s'agit là d'une évidence que les recherches des deux dernières décennies ont largement contribué à dévoiler. Pour le christianisme, seul monothéisme à les avoir acceptées, les images de la divinité assurent la communication avec leur prototype; véritables «présentifications », elles accomplissent au milieu des chrétiens toutes sortes de prodiges ${ }^{1}$. Les images qui ne figurent pas le Christ, la Vierge ou les saints peuvent également posséder un pouvoir: c'est le cas des enseignes médiévales aux sujets les plus variés, étudiées par Denis Bruna, ainsi que des sculptures opérant comme des «talismans de pierre», dont Fulvio Cervini a dressé un riche catalogue ${ }^{2}$.

Dans la présente étude, nous nous proposons de revenir sur ce dossier en nous focalisant plus particulièrement sur des images agissantes intégrées dans le décor monumental extérieur des édifices du Moyen Âge, qui ne figurent pas la divinité. Après avoir considéré quelques images faisant l'objet d'incantation magique ou témoignant d'une pratique rituelle de fondation ou de protection, nous nous pencherons sur les sculptures dont les propriétés semblent venir uniquement du sujet représenté, sans que l'intervention d'un «magicien» ne soit nécessaire. Nous essayerons ensuite d'analyser le fonctionnement de ces images agissantes et de mettre en exergue les principes qui garantissent leur efficacité. Dans le cadre d'un

1. Jean-Claude Schmitt, Le corps des images: essais sur la culture visuelle au Moyen Âge, Paris, Gallimard, 2002. Parmi les nombreuses études de JeanMarie Sansterre, nous nous limitons à signaler «L'image blessée, l'image souffrante: quelques récits de miracles entre Orient et Occident $\left(\mathrm{VI}^{\mathrm{e}}-\mathrm{XII}^{\mathrm{e}}\right.$ siècle)», Les images dans les sociétés médiévales. Pour une histoire comparée. Actes du colloque international (Rome, 19-20 juin 1998), dir. Jean-Marie Sansterre et Jean-Claude Schmitt, Bruxelles, 1999, p. 113-130; «Miracles et images: les relations entre l'image et le prototype céleste d'après quelques récits des $\mathrm{X}^{\mathrm{e}}$-XIII ${ }^{\mathrm{e}}$ siècles", Problèmes d'histoire des religions, 19 (2009), p. 47-57. Jérôme Baschet, L'iconographie médiévale, Paris, Gallimard, 2008 (notamment p. 44-60, sur l'efficacité des images). La performance des images, dir. Alain Dierkens, Gil Bartholeyns et Thomas Golsenne, Bruxelles, Université de Bruxelles, 2009.

2. Denis Bruna, Enseignes de plomb et autres menues chosettes du Moyen Âge, Paris, Léopard d'or, 2006; Fulvio Cervini, «Talismani di pietra: sculture apotropaiche nelle fonti medievali», Lares, 67 (2001), p. 165-188; Id., «Pietre portentose, ovvero come i medievali vedevano le sculture apotropaiche», Metodo della ricerca e ricerca del metodo: storia, arte, musica a confronto, dir. Benedetto Vetere, Galatina, Congedo, 2009, p. 129-150. 
volume consacré à l' «objet rituel», il sera utile de nous interroger sur les rapports que l'on peut établir entre ces images, les rites, notamment ceux de passage ${ }^{3}$, et la magie médiévale. Face aux différentes définitions que les concepts de «rite» et de «magie» ont reçues de la part des anthropologues, sociologues et historiens, il ne sera pas question de prendre parti pour l'une ou l'autre méthode, mais de montrer de quelle manière le fonctionnement de ces images peut s'adapter ou non à des modèles interprétatifs élaborés pour les rites et la magie.

\section{Des images et Des Rites}

\section{Des images comme supports d'incantation}

Les images sont parmi les supports privilégiés des enchantements des magiciens au Moyen Âge. Nicolas Weill-Parot a bien mis en évidence le rôle des «images astrologiques», capables de capter et d'orienter l'influx des astres, dans la magie occidentale ${ }^{4}$. D'autres sources éparses relatent les propriétés d'objets figurés n'ayant pas de liens apparents avec les astres.

De nombreux témoignages concernent des incantations à des images faisant partie du décor monumental. Ainsi, certains auteurs (parmi lesquels Jean de Salisbury, Conrad de Querfurt, Gervais de Tilbury et Vincent de Beauvais) attestent qu'au-dessus d'une porte de la ville de Naples était placée une mouche de bronze, grosse comme une grenouille, à qui Virgile en personne, le poète de l'Antiquité, aurait attribué le pouvoir d'éloigner les vraies mouches, nombreuses dans les eaux tout autour de la ville ${ }^{5}$. Mais Naples conservait beaucoup d'autres traces de l'activité magique de Virgile. Gervais de Tilbury atteste avoir vérifié personnellement l'efficacité de deux têtes de marbre placées par le poète à droite et à gauche d'une autre porte de la ville: elles procuraient à tout

3. Arnold van Gennep, Les rites de passage. Étude systématiques des rites, Paris, 1969 (réimpr. Paris, 1981; voir p. 19-33, sur les passages des seuils).

4. Nicolas Weill-Parot, Les «images astrologiques» au Moyen Âge et à la Renaissance. Spéculations intellectuelles et pratiques magiques (XII ${ }^{e}-\mathrm{XV}^{e}$ siècles), Paris, Honoré Champion, 2002.

5. N. Weill-Parot, Les «images astrologiques», p. 147-149. 
arrivant, selon le côté par lequel il passait, une bonne ou une mauvaise fortune ${ }^{6}$.

Selon plusieurs sources du $\mathrm{Xv}^{\mathrm{e}}$ siècle, le magicien et astrologue Guido Bonatti aurait accompli entre 1231 et 1298 de nombreux rites en se servant des images. Ainsi, afin de protéger la ville de Forlì, il aurait placé un bucrane en pierre sur le côté de l'église SainteCroix. La Chronique de Giovanni de Pedrino relate que sa rupture accidentelle en 1429 sema la panique auprès de la population persuadée de la valeur protectrice de cette image-objet ${ }^{7}$.

À ces exemples d'enchantements opérés sur des images monumentales, on pourra ajouter celui effectué par un clerc nécromancien appelé Flocars, cité par le Roman d'Abladane, écrit vers 1260. Parmi d'autres prodiges, celui-ci dota la porte de la ville d'une statue de la Vierge (invisible car renfermée dans un caisson qui devait s'ouvrir uniquement pour le seigneur de la ville) et de deux gargouilles: celles-ci étaient censées jeter un poison sur tous ceux qui essayaient de s'emparer de la cité, et déverser de l'or et de l'argent au passage du souverain légitime ${ }^{8}$.

Toutes les images citées ci-dessus se chargent de pouvoirs grâce à l'intervention d'un magicien. Virgile, Guido Bonatti, Flocars les fabriquent ou, plus généralement, les placent à des endroits stratégiques. Elles font sans doute l'objet d'un rite, que les sources ne nous décrivent pourtant pas. Ces textes ne sont pas en effet des traités de magie et, dans ce cadre, la mention de l'intervention du magicien suffisait probablement à prouver l'efficacité du rite.

6. Texte latin dans Domenico Comparetti, Virgilio nel Medioevo, FlorenceRome, 1896, $2^{\mathrm{e}}$ vol., p. 187-191. Pour la traduction française, cf. Annie Duchesne, Le Livre des Merveilles, Paris, Les Belles-Lettres, 1992, p. 31-32 (pour les autres enchantements de Virgile à Naples, p. 28-34). Pour la réputation de Virgile au Moyen Âge, voir J. W. Spargo, Virgil the Necromancer, Cambridge (Mass.), Harvard University Press, 1934.

7. Giovanni de Maître Pedrino Depintore, Cronica del suo tempo, I, éd. Gino Borghezio et Marco Vattasso, Rome, BAV, 1929, p. 164. Pour celle-ci et pour les autres sources du Xv $\mathrm{Xv}^{\mathrm{e}}$ siècle sur Guido Bonatti, voir F. Cervini, «Talismani», p. 165-168. Sur Guido Bonatti, voir aussi Cesare Vasoli, «Guido Bonatti», Dizionario biografico degli Italiani, 11 (1969), p. 603-608; N. Weill-Parot, Les «images astrologiques», p. 391-394 (sur son usage des «images astrologiques»). Sur la magie médiévale, voir Jean-Patrice Boudet, Entre science et nigromance: astrologie, divination et magie dans l'Occident médiéval (XII ${ }^{e}-V^{e}$ siècles), Paris, Publications de la Sorbonne, 2006.

8. Le Roman d'Abladane, éd. Giovanni Palumbo, Paris, Honoré Champion, 2011, chap. 27-28, p. 78-79. 
D'ailleurs, les procédés d'enchantement relèvent par définition de l'occulte et doivent rester cachés aux non-initiés.

\section{Des images comme traces d'un rite}

En plus de servir de support à une incantation, l'image peut être la trace d'une pratique ou d'un rite. Ce principe est d'origine antique: les bucranes, motif décoratif très commun dans l'Antiquité, sont probablement la version en pierre des têtes de taureaux ou de bœufs enguirlandés sacrifiés aux dieux païens ${ }^{9}$.

Jen'ai pu repérer qu' un seul cas d'image médiévale de ce type. Dans la petite église romane de San Pantaléon de Losa (Burgos) (fig. 1), les archivoltes des portails et des fenêtres présentent des personnages sculptés littéralement emmurés dans la pierre, dont on n'aperçoit plus que la tête et les jambes. De manière perspicace et convaincante, Fulvio Cervini a relié ces images à la pratique médiévale d'intégrer des corps humains, ou des restes de ceux-ci, dans les murs ou dans les éléments de support des édifices: au Moyen Âge, et jusqu'à la Renaissance, des tombes étaient parfois intégrées dans les piliers des églises et des reliques de saints étaient insérées dans les parois ou dans les chapiteaux. Il s'agit là d'une manière d'assurer la protection de l'édifice par les défunts, dont les saints sont une catégorie «très spéciale $»^{10}$ : leurs corps se fondent dans les murs et contribuent à défendre et à soutenir l'église. Il existe d'innombrables variantes de cette pratique de fortification des bâtiments par les défunts: ainsi, des récits folkloriques de toute époque attestent que des sacrifices humains étaient accomplis lors de la construction d'édifices, surtout des ponts, dans différentes civilisations ${ }^{11}$.

9. Sur l'évolution des formes du bucrane dans l'Antiquité, voir Adolf Ernst Napp, Bukranion und Guirlande. Beiträge zur Entwicklungsgeschichte der hellenistischen und römischen Dekorationskunst, Heidelberg, 1933.

10. La formule est célèbre depuis les travaux de Peter Brown, The Cult of the Saints: Its Rise and Function in Latin Christianity, Chicago, Londres, 1981.

11. Fulvio Cervini, «Mura cementate col sangue: un percorso medievale tra riti di fondazione e reimpieghi anomali», Medioevo. Immagine e memoria. Atti del convegno internazionale di studi (Parme, 23-28 septembre 2008), dir. Arturo Carlo Quintavalle, Milan, Electa, 2009, p. 325-336. Sur l'intégration des défunts à l'édifice de culte, voir également Michel Lauwers, «Des "pierres vivantes". Construction d'églises et construction sociale dans l'Occident médiéval», Matérialité et immatérialité dans l'Église au Moyen Âge. Actes du colloque international (Bucarest, 22-23 octobre 2010), Bucarest 2012, p. 359-378. 
Les personnages emmurés dans les archivoltes de San Pantaleón de Losa renvoient donc très probablement à des défunts protecteurs de l'église, sans qu'il soit possible de déterminer leur identité. Ces sculptures exercent probablement le même pouvoir que les morts qu'elles représentent: intégrées dans l'archivolte, elles soutiennent l'édifice; placées au-dessus du portail et des fenêtres, elles en protègent les entrées.

\section{LE POUVOIR INTRINSÈQUE DE CERTAINS SUJETS}

Si les images traitées jusqu'à présent ont des relations avec des pratiques accomplies par des hommes, de nombreux autres éléments figurés du décor monumental semblent au contraire tirer leur pouvoir du sujet qu'ils représentent, sans que l'intervention d'un magicien ne soit requise. Nous nous pencherons ici sur quelques thèmes très récurrents dans la sculpture monumentale médiévale, qui semblent avoir des propriétés particulières: il s'agit des statues de lions, du décor à rinceaux habités et des têtes grimaçantes ou monstrueuses, notamment quand elles apparaissent sur les gargouilles.

\section{Le lion, fauve polysémique}

Les lions sont probablement les animaux les plus représentés sur les seuils des bâtiments. À toute époque et dans différents horizons géographiques, des villes et des édifices sont dotés de portes «aux lions». Au Moyen Âge, au IX ${ }^{\mathrm{e}}$ siècle, des têtes léonines apparaissent sur les heurtoirs de quelques battants, avant que le fauve entier ne soit sculpté en haut-relief sur les façades de certains édifices du début du $\mathrm{XI}^{\mathrm{e}}$ siècle. Des statues de lions en ronde bosse, antiques ou médiévales, commencent à être placées de part et d'autre des seuils des églises, à partir du milieu du $\mathrm{XI}^{\mathrm{e}}$ siècle ${ }^{12}$.

Le rôle le plus commun de ces statues semble être celui de gardiens des entrées. Une inscription autrefois placée à côté d'un

12. Pour plus d'exemples et pour des références plus précises à ce sujet, je me permets de renvoyer à mon article «Têtes, lions et attributs sexuels: survivances et évolutions de l'usage apotropaïque des images de l'Antiquité au Moyen Âge», Cahiers de Saint-Michel de Cuxa, 39 (2008), p. 209-221. 
de ces félins sur la façade de la cathédrale de Verdun, consacrée en 1147, affirme: "Le lion est un gardien parce qu'il dort les yeux ouverts: c'est pour cette raison qu'on le place devant les portes» (EST LEO SED CUSTOS OCULIS QUIA DORMIT APERTIS TEMPLORUM IDCIRCO PONITUR ANTE FORAS). La croyance remonte à une légende, rapportée par Isidore de Séville et Raban Maur, selon laquelle les lions dormaient les yeux ouverts, qualité qui en faisait d'excellents gardiens ${ }^{13}$.

La fonction de sentinelle attribuée à cet animal est encore évoquée au XIII ${ }^{\mathrm{e}}$ siècle par Georges de Gallipoli, lorsqu'il célèbre un portail du palais épiscopal de la même ville où prennent place deux lions et l'aigle impérial. Le poète affirme qu'il n'y aura besoin d'aucune serrure pour cette porte, car ces trois gardiens veillent et épouvantent ceux qui tentent de s'y introduire ${ }^{14}$.

La miniature d'une bible parisienne du XIV siècle figurant le trône de Salomon est également très explicite. Deux lions, disposés de part et d'autre de la porte du paradis, en maintiennent les vantaux ouverts et protègent l'entrée; des inscriptions les qualifient respectivement de TERROR DEMONUM et TERROR INIMICORUM ${ }^{15}$.

Dès l'Antiquité, le lion peut avoir une connotation funéraire: bête anthropophage, il dévore les hommes, tout comme la mort ${ }^{16}$. Dans la vision chrétienne, il peut également renvoyer à l'au-delà : comme Waldemar Deonna l'a jadis montré, dans nombre de passages

13. Sur l'inscription de Verdun, aujourd'hui perdue, et sur son interprétation, voir Robert Favreau, «Le thème iconographique du lion dans les inscriptions médiévales», Comptes rendus des séances de l'Académie des Inscriptions et Belles-Lettres, 135 (1991), p. 613-636, p. 623.

14. Versus Georgii Chartophylacis Callipolis, X, vv. 1-7, Poeti bizantini di Terra d'Otranto nel secolo XIII, éd. Marcello Gigante, Galatina, Congedo, 1985, p. 186-187 (cité par F. Cervini, «Talismani», p. 138).

15. Fribourg, Bibliothèque cantonale et universitaire, ms. L 75 II, fol. 1 (1334-1336). Isa Ragusa, «Terror Demonum et Terror Inimicorum: The Two Lions of the Throne of Salomon and the Open Door of Paradise», Zeitschrift für Kunstgeschichte, 40 (1977), p. 93-114.

16. Jacques Dumont, Les animaux dans l'antiquité grecque, Paris, L'Harmattan, 2001, p. 164-165; Cornelius Vermeule, «Funerary Animals, 450300 B. C.», American Journal of Archaeology, 76 (1972), p. $49-59$ (en part. sur les lions : p. 49-56); Fraser Hunter, Funerary Lions in Roman Provincial Art, dans Romanisation und Resistenz in Plastik, Architektur und Inschriften der Provinzen des Imperium Romanum. Neue Funde und Forschungen, éd. Peter Noelke, Friederike Naumann-Steckner et Beate Schneider, Mayence, von Zabern, 2003, p. 59-65. 
bibliques, il est assimilé aux puissances infernales ${ }^{17}$. Les statues médiévales montrant ces félins en train de dévorer des hommes miniaturisés se réfèrent probablement à cet aspect: les corps des victimes sont souvent nus, les membres désarticulés, comme les âmes des damnés figurées dans certaines représentations de l'Enfer (fig. 2) ${ }^{18}$. Sur les seuils des églises, ces hommes incarnent vraisemblablement le sort des pécheurs qui ne sont pas dignes d'entrer dans l'édifice.

Dans sa polysémie, le lion peut également constituer une figure du Christ. Le célèbre tympan du portail de Jaca, en Aragon, en offre l'un des exemples le plus explicites. Ici, deux lions affrontés flanquent un grand chrisme: l'un écrase un homme qui agrippe un serpent, tandis que son vis-à-vis se tient debout sur un ours et un basilic. L'inscription de gauche, rapprochant le lion du Christ, affirme: «Le lion sait épargner celui qui se prosterne, et le Christ [sait épargner] le pénitent» (PARCERE STERNENTI LEO SCIT, Xristusque PETENTI); l'inscription de droite, où l'assimilation du félin au Christ est implicite, mais claire, affirme: «Le lion fort terrasse l'empire de la mort» (IMPERIUM MORTIS CONCULCANS EST LEO FORTIS $)^{19}$. Sur ce tympan, inscriptions et images invitent les Chrétiens qui entrent dans l'église à réfléchir sur la nécessité de la pénitence et de la soumission au Christ, seule voie pour échapper à la mort de l'âme.

La christianisation du lion et sa relation avec le trépas interviennent dans une autre sculpture du $\mathrm{XII}^{\mathrm{e}}$ siècle, à Rome. Deux statues encadrent en effet le portail de San Lorenzo in Lucina (fig. 3) : celle de gauche figure le fauve avec son petit entre les pattes, tandis que celle de droite (fig. 4) accueille au même emplacement un enfant. Le lion de gauche semble mettre en image une légende tirée du bestiaire, selon laquelle les lionceaux, mort-nés, étaient ramenés à la vie par le souffle de leur père, trois jours après leur naissance.

17. Waldemar Deonna, «Salva me de ore leonis», Revue Belge de Philologie et d'Histoire, 28 (1950), p. 479-511, p. 486-493.

18. Voir par exemple les damnés dans le manuscrit du Beatus du milieu du $\mathrm{X}^{\mathrm{e}}$ siècle conservé à New York (Pierpont Morgan Library, ms. 644, f. 220r); repr. dans Yves Christe, Jugements derniers, Saint-Léger-Vauban, 2000, p. 59-60, fig. 18.

19. Sur ce portail, Susan Havens Caldwell, «Penance Baptism, Apocalypse: The Easter Context of Jaca Cathedral's West Tympanum », Art History, 3 (1980), p. 25-40. 
Au Moyen Âge, ce récit permettait d'identifier le lion avec Dieu et le Christ à la fois, ressuscité par le Père après trois jours dans le sépulcre ${ }^{20}$. Le nourrisson placé de manière identique entre les pattes de son congénère est manifestement le pendant du Christlionceau. Or, l'enfant est normalement la forme sous laquelle est alors représentée l'âme humaine, précisément dans les scènes de mort ou de conception. Il est donc possible que ces images fassent allusion à la mort et à la résurrection de ceux qui franchissent le seuil: l'entrée dans l'église est assimilée au début d'une nouvelle $\mathrm{vie}^{21}$.

Ce choix d'exemples montre la pluralité sémantique des statues de lion placées au seuil des églises. Elles peuvent être des objets purement apotropaïques (comme le sont les statues de lions antiques remployées, surtout là où la bête n'a pas de proie) ou fonctionner de manière plus complexe. Dévorant des corps humains nus, elles peuvent mettre en scène la mort des âmes en Enfer. À ce titre, on peut voir dans ces images une fonction d'admonestation, particulièrement appropriée aux portails d'une église, comparable à la figuration des châtiments des damnés dans les enfers des tympans romans $^{22}$. À Jaca et à San Lorenzo in Lucina, les images du Christlion ont également une fonction d'admonestation: dans les deux cas, seuls ceux qui se placent sous le signe du Christ peuvent espérer vaincre la mort et renaitre à une vie nouvelle. Que ce message soit figuré en contexte de seuil revêt une très grande importance, car il rapproche la porte de l'église de l'entrée vers l'au-delà.

20. Parmi les auteurs qui opèrent ce rapprochement, cf. Raban Maur, De rerum naturis, lib. VIII, cap. I; PL 111, col. 218B; Alcuin, Commentaria in Apocalipsin, lib. II, cap. 4, vers. 7; PL 100, 1118C.

21. J'ai déjà eu l'occasion de me pencher sur les lions de San Lorenzo in Lucina, dans A. Trivellone, «Têtes», p. 218. Dans cette étude, j'affirmais que les deux lions forment un couple mâle/femelle, mais, après une observation plus attentive, rien ne permet de l'affirmer. Les deux bêtes relèvent plutôt de deux types différents de représentations léonines de l'Antiquité: le félin de gauche correspond au type «romain», alors que celui de droite est de type «égyptien». Je remercie Rolf Stucky de m'avoir apporté cette précision.

22. On rappellera à ce propos le cas célèbre du tympan de Conques où une effrayante représentation de l'enfer est accompagnée d'une inscription qui rappelle aux pécheurs le sort qui les attend s'ils ne changent pas de comportement: o PECCATORES TRANSMUTETIS NISI MORES IUDICIUM DURUM VOBIS SCITOTE FUTURUM ( «Pécheurs, si vous ne réformez pas vos mœurs, sachez que vous subirez un jugement redoutable»). 


\section{Les vertus cathartiques des rinceaux habités}

Un autre motif très répandu dans la sculpture monumentale du Moyen Âge, susceptible d'avoir une valeur agissante, est celui des «rinceaux habités», entrelacs de végétation qui abritent des hommes, des bêtes, des hybrides ou des monstres. Les formes médiévales de ce thème sont une élaboration du motif antique des putti dans les rinceaux de vigne ou d'acanthe, utilisé dans des contextes funéraires, surtout à l'époque paléochrétienne ${ }^{23}$.

Longtemps considérés comme de simples motifs décoratifs, des études de cas ont décelé dans ce type de figuration des implications riches de sens. Ainsi, en 1995, Jean-Pierre Caillet a mené une importante analyse des rinceaux couvrant de nombreuses pièces de mobilier liturgique, de la fin du $\mathrm{X}^{\mathrm{e}}$ au début du XIII ${ }^{\mathrm{e}}$ siècle $^{24}$. Pour la sculpture monumentale, d'autres études ont porté sur le linteau du Saint Sépulcre de Jérusalem (XII ${ }^{\mathrm{e}}$ siècle $)^{25}$, les portails de l'église de San Michele della Chiusa (Piémont) ${ }^{26}$, les colonnettes de façade à Saint-Denis à Paris ${ }^{27}$ et les portails d'un groupe d'églises dans les Abruzzes (fig. 5) ${ }^{28}$. En dépit des variantes, tous ces rinceaux semblent être une métaphore moralisante de la vie terrestre où dominent les péchés. Les rinceaux seraient une évocation de la silva, caractérisée par la riche végétation et les bêtes sauvages: la «forêt» serait donc entendue comme une allusion au monde post-

23. Sur ce motif dans l'art classique, cf. John B. Perkins, Jocelyn M. C. Toynbee, «Peopled Scrolls: A Hellenistic Motif in Imperial Art», Papers of the British School at Rome, n. s., 5 (1950), p. 1-43.

24. Jean-Pierre Caillet, «Et magnae silvae creverunt... Observations sur le thème du rinceau peuplé dans l'orfèvrerie et l'ivoirerie liturgiques aux époques ottonienne et romane», Cahiers de Civilisation médiévale, 38 (1995), p. 23-33.

25. Bianca Kühnel, «Der Rankenfries am Portal der Grabeskirche zu Jerusalem und die Romanische Skulptur in den Abruzzen», Arte medievale, $2^{\mathrm{e}}$ série, 1 (1987), p. 87-121.

26. Christine Verzar Bornstein, Die romanische Skulpturen der Abtei Sagra di San Michele. Studien zu Meister Nicolaus und zur «Scuola di Piacenza», Langenthal, Merkur, 1968, p. 69-71.

27. Jean-Claude Bonne, «Le végétalisme de l'art roman: naturalité et sacralité», Le monde végétal. Médecine, botanique, symbolique, dir. Agostino Paravicini Bagliani, Florence, Sismel, 2009, p. 95-120.

28. Je me permets à ce propos de renvoyer à mon étude «Les rinceaux habités des portails de la Marsica (Abruzzes, Italie): purification et élévation au seuil de l'église», Thèmes religieux et thèmes profanes dans l'image médiévale: transferts, emprunts, oppositions, Actes du colloque international (Paris, 23-24 mai 2011), dir. Christian Heck, Turnhout, Brepols, 2013, p. 125-142. 
adamique, où l'homme, à cause de son péché originel, a perdu la domination sur la «nature». Dans ce cadre, éviter l'agression des animaux sauvages revient à vaincre ses propres passions bestiales, à l'origine du péché: la silva devient le lieu des épreuves qui mènent à un état plus élevé de perfection et au salut.

Tout comme les statues de lions, les rinceaux habités confèrent un sens profond au passage du seuil, en favorisant, telle une silva, la purification et l'élévation de ceux qui s'apprêtent à entrer dans l'église.

\section{Les têtes grimaçantes et les gargouilles}

Souvent liées aux entrées des édifices, les têtes animales et les protomes grimaçantes sont susceptibles de recevoir une fonction protectrice. Pour le Moyen Âge, le témoignage le plus explicite à ce sujet vient du Roman d'Ysengrin, écrit au milieu du XII ${ }^{\mathrm{e}}$ siècle. Ysengrin lui-même y décrit la pratique centenaire d'accrocher au mur en dehors de la porte la gueule d'animaux sauvages, normalement de loups, comme avertissements sinistres à l'encontre des hommes animés de mauvaises intentions et des esprits diaboliques ${ }^{29}$.

C'est probablement en vertu de leur pouvoir apotropaïque que les figures de proue des bateaux médiévaux, y compris ceux de guerre, reproduisent des têtes humaines, animales ou monstrueuses, souvent grimaçant ou tirant la langue ${ }^{30}$.

Les gargouilles, sculptures fortement saillantes ayant souvent un rôle de canalisation, prennent fréquemment la forme de têtes grimaçantes ou monstrueuses (fig. 6). Si elles peuvent avoir une finalité apotropaïque, Pierre-Olivier Dittmar et Jean-Pierre Ravaux ont montré que leurs fonctions sont en fait plus variées. En étudiant les gargouilles en pierre de Notre-Dame de l'Épine (Marne), ils ont établi que l'ensemble de ces sculptures construit un discours cohérent, visible tout particulièrement depuis le cimetière contigu à l'église: dans ce cadre, elles admonestent les pécheurs et préfigurent

29. Ysengrimus, éd. Jill Mann, Leiden, Brill, 1987, p. 397 (cité par Michael Camille, «Mouth and Meanings: Towards an Anti-Iconography of Medieval Art», Iconography at the Crossroads, dir. Brendan Cassidy, Princeton, Princeton University Press, 1993, p. 43-58, p. 52).

30. Sur ces questions, je me permets de renvoyer à A. Trivellone, «Têtes», p. 211-215. 
les châtiments de l'au-delà, en accompagnant l'observateur dans un parcours qui mène jusqu'à l'enfer ${ }^{31}$.

Lions, rinceaux, têtes grimaçantes ne constituent que quelques exemples de sujets agissants. D'autres seraient susceptibles d'enrichir le corpus. Parmi ceux-ci, les personnages exhibant leur sexe, notamment les sculptures de femmes appelées communément SheelaNa-Gig, pourraient occuper une place tout à fait intéressante. Malgré des travaux pionniers ${ }^{32}$, ces sculptures attendent toujours des analyses contextuelles poussées qui permettront de les prendre éventuellement en compte dans une étude générale des motifs agissants.

\section{FONCTIONNEMENT ET PRINCIPES D'EFFICACITÉ DES IMAGES MONUMENTALES AGISSANTES}

Les quelques images considérées dans les pages qui précèdent sont très hétérogènes: leur seul point commun est de posséder un pouvoir. Parfois, leurs propriétés sont attestées par des sources écrites (chroniques, poésies, inscriptions, etc.), dans d'autres cas ce n'est que l'étude croisée des sujets représentés et de leur contexte qui laisse entrevoir leurs vertus. Il s'agit maintenant de porter un regard synthétique sur ce corpus d'exemples, afin d'examiner leur fonctionnement et les principes de leur efficacité.

\section{L'action des images sur l'espace et sur les hommes}

Les images agissantes peuvent avoir un rayon d'action variable. Ainsi, le bucrane de Guido Bonatti est susceptible de protéger toute la ville depuis son emplacement sur un flanc de l'église de Forlì ; ailleurs les gargouilles protègent les édifices en usant de leur pouvoir répulsif, renvoyant le mal vers l'extérieur. La majorité de ces images se trouve à un endroit stratégique, à savoir en relation avec une ouverture, le plus souvent un seuil: c'est à partir de là que leur action s'adresse plus particulièrement aux hommes. Leurs

31. Pierre-Olivier Dittmar et Jean-Pierre Ravaux, «Signification et valeur d'usage des gargouilles: le cas de Notre-Dame de l'Épine», Études marnaises, 123 (2008), p. 39-80.

32. Barbara Freitag, Sheela-Na-Gigs. Unravelling an Enigma, Londres-New York, 2004. 
buts sont multiples: elles peuvent admonester, effrayer, punir les hommes ou les empêcher d'accomplir de mauvaises actions. Mais elles peuvent aussi honorer les souverains qui franchissent la porte, comme les gargouilles enchantées de Flocars, ou porter bonheur aux passants, comme l'une des têtes placées par Virgile à l'entrée de la ville de Naples, ou encore accompagner et purifier l'homme lors du franchissement du seuil d'un édifice sacré, assimilé à une mort suivie d'une résurrection.

Parfois, la seule présence des images suffit à leur efficacité. La Vierge de Flocars, renfermée dans un caisson, ne devait pas être vue: elle agissait à l'insu des hommes. D'autres fois, la vision des images, comme par exemple celle des lions dévorant des proies humaines, servait probablement à épouvanter ou à émouvoir.

Le pouvoir des images est parfois capable de les animer et de les faire littéralement bouger. C'est le fait surtout des gargouilles. Pendant les jours de pluie, ces dernières devaient sembler vivantes: il était alors facile de les imaginer en train de cracher, vomir ou expulser par d'autres orifices corporels des liquides, que l'on pouvait imaginer mauvais ou bons, selon les cas. Les gargouilles d'Amiens, enchantées par Flocars, fonctionnent selon ce principe: elles crachent du poison sur les ennemis, et de l'or et de l'argent pour le seigneur de la ville. Même des gargouilles dépourvues de toute fonction de canalisation peuvent s'animer. C'est ce qui se passe à Dijon, quand, selon le dominicain Étienne de Bourbon, un usurier allait se marier dans l'église Notre-Dame. Arrivé avec sa future épouse devant la façade, «un autre usurier de pierre, sculpté avec une bourse, au-dessus de la porte» (alius usurarius lapideus qui erat sculptus cum bursa supra portam) jeta son aumônière sur le futur mari qui mourut sur le coup ${ }^{33}$.

Dans ces cas, les gargouilles cherchent le contact avec ceux qui entrent dans leur rayon d'action, par leurs sécrétions ou par des objets qu'elles lancent. Il est possible que, dans d'autres cas, ce fussent les hommes qui touchaient les images agissantes. C'est ce que laissent penser les pattes du lion de gauche de San Lorenzo in

33. Sur la capacité des gargouilles de s'animer, voir P.-O. Dittmar, J.-P. Ravaux, «Signification», p. 43. L'épisode de la gargouille de Dijon, relaté par les deux chercheurs, est raconté par Étienne de Bourbon, Tractatus de diversis materiis predicabilibus, éd. Jacques Berlioz et Jean-Luc Eichenlaub, Turnhout, Brepols, 2002, livre I, VII, 1. 285-295, p. 280. 
Lucina, ainsi que le corps de son lionceau : ils semblent usés comme certaines statues de saints frottées par les fidèles pendant de très longues périodes (fig. 3).

\section{Les principes de l'efficacité}

Les exemples choisis permettent d'identifier certains éléments qui semblent présider à l'efficacité d'une image. Le principe le plus simple est l'intervention d'un artifex ou magicien, capable par son rite d'infuser un pouvoir à l'objet. Il est intéressant de remarquer que l'efficacité semble subordonnée à l'intégrité de ce dernier et que sa rupture peut provoquer la perte des pouvoirs: Conrad de Querfurt et Gervais de Tilbury soulignent tous deux que la mouche de Virgile doit rester intacte et à sa place pour conserver ses propriétés $^{34}$; la Chronique de Giovanni de Pedrino relate, de son côté, que la cassure du bucrane de Guido Bonatti en 1429 a causé la panique auprès de la population qui croyait en son efficacité.

À défaut de l'intervention d'un magicien, le sujet représenté semble pouvoir assurer à lui seul l'efficacité de l'image. Les images agissantes dont nous avons traité ressemblent par leur forme à des êtres qui existent en «nature». La relation au prototype joue un rôle important: ainsi, puisque les lions dorment les yeux ouverts, leurs statues aussi; la forêt ayant des propriétés cathartiques, sa représentation sur les seuils peut accomplir la même fonction. Le pouvoir de ces images se fonde donc sur la similitudo, un principe que l'on retrouve régulièrement dans les traités de magie ${ }^{35}$. Il est possible, par exemple, d'établir des parallèles entre nos images et celles évoquées dans le célèbre traité Picatrix, que Nicolas WeillParot appelle «corporéiformes » ou «naturiformes», du fait de leur ressemblance avec des êtres existant en «nature $»^{36}$. Appliquant à

34. Voir D. Comparetti, Virgilio, p. 185 (sur Conrad) et p. 187 (sur Gervais).

35. Loin d'être une particularité médiévale, la similitudo est un élément important de tout acte magique: James Georges Frazer créa à ce propos le concept de «magie sympathique». Très critiquée au cours du $\mathrm{xx}^{\mathrm{e}}$ siècle, sa théorie semble garder, dans notre cas, sa valeur heuristique; cf. James Georges Frazer, Le rameau d'or, éd. Nicole Belmont et Michel Izard, 4 vol., Paris, Robert Laffont, 1981-1984 (sur la réception de l'œuvre au $\mathrm{Xx}^{\mathrm{e}}$ siècle, voir Michel Izard, «Introduction. Le roi magicien dans la société primitive», vol. 1, p. XXXI-XLVIII, p. XLI-XLVII).

36. Nicolas Weill-Parot, «Images corporéiformes et similitudo dans le Picatrix et dans le monde latin médiéval», Images et magie. Picatrix entre Orient et Occident, dir. Jean-Patrice Boudet, Anna Caiozzo, Nicolas Weill-Parot, Paris, 
nos sculptures la classification proposée par l'historien, on pourra affirmer que certaines sont des images «corporéiformes de cible», d'autres sont «corporéiformes de source», alors que d'autres artefacts rentrent simultanément dans les deux types. Les images «corporéiformes de cible» reproduisent l'objet de l'effet magique: c'est le cas de la mouche de Virgile, qui a la même forme que les insectes que l'on veut éloigner de la ville de Naples. Les images «corporéiformes de source» figurent l'objet dont le pouvoir tire son origine: c'est le cas des images des défunts de San Pantaleón de Losa, du bucrane de Guido Bonatti ou des lions (dépourvus de proie) à l'entrée des églises. Enfin, d'autres images mettent simultanément en scène sources et cibles du pouvoir: c'est le cas des rinceaux habités, qui représentent la silva ainsi que les hommes qui se purifient en la traversant, et des lions avec leurs "proies», qu'elles soient dévorées ou qu'elles soient épargnées par le fauve. Nous ne pouvons toutefois pas assurer que toutes les images considérées entrent dans cette grille de lecture: Gervais de Tilbury nous dit trop peu, par exemple, des deux têtes placées par Virgile de part et d'autre de la porte de Naples pour qu'on puisse hasarder une classification quelconque ${ }^{37}$.

Au-delà des liens de similitudo, d'autres facteurs semblent avoir un rôle sur l'efficacité des images. Ainsi, en premier lieu, on remarque que la représentation d'animaux et de lieux «naturels» semble particulièrement efficace. L'image semble alors s'approprier des pouvoirs sauvages par une forme de «domestication». Les lions, les têtes animales monstrueuses grimaçantes - l'existence réelle de certains monstres n'étant pas remise en cause au Moyen Âge - ou encore la silva, rentrent dans cette catégorie ${ }^{38}$.

Dans plusieurs cas, il semblerait toutefois que cette évocation de la «nature» passe à travers une reprise de l'antique. Les sculptures

Honoré Champion, 2011, p. 117-136. Pour la version française de célèbre traité de magie, traduit en 1256 de l'arabe en castillan, voir Picatrix. Un traité de magie médiéval, éd. Béatrice Bakhouche, Frédéric Fauquier et Brigitte Pérez-Jean, Turnhout, Brepols, 2003.

37. Cf. supra, note 6.

38. Jean-Claude Schmitt a déjà remarqué ce phénomène et observe que «les clercs s'emparent de cette force vitale pour se doter d'un surcroît de sacralité»; cf. Jean-Claude Schmitt, «Les images et le sacré», La performance, p. 29-46, p. 34. Sur la performativité des images zoomorphes au Moyen Âge, voir aussi PierreOlivier Dittmar, «Performances symboliques et non-symboliques des images animales », La performance, p. 59-70. 
de lions flanquant les entrées médiévales sont de véritables remplois de statues funéraires grecques et romaines ou s'en inspirent; bien qu'élaborés au Moyen Âge, les rinceaux habités ont aussi une origine antique. Il en est de même pour le motif du bucrane, dont un exemplaire, remploi ou artefact médiéval, est l'objet de l'incantation de Guido Bonatti. La relation avec l'Antiquité est encore explicite quand c'est Virgile même qui est censé avoir opéré des prodiges. De fait, les sculptures grecques et romaines étaient souvent censées posséder des vertus magiques au Moyen Âge ${ }^{39}$.

L'aura dont les Antiques jouissaient dans la perception médiévale est probablement à rapprocher du culte des ancêtres ou des défunts. Par ailleurs, la relation avec les morts constitue un autre aspect fondamental de la magie médiévale, souvent associée à la nécromancie. Dans nos images, cette relation aux morts est évidente dans les sculptures de San Pantaleón de Losa et revient dans les gargouilles d'Amiens, dont le venin est «destrempé»par Flocars à partir des «tombiaux $»^{40}$. Virgile, comme nous l'avons $\mathrm{vu}$, jouissait d'une réputation de nécromancien: c'est bien pour cela que Dante, dans sa Divine Comédie, le choisit comme guide à travers l'au-delà. Il est d'ailleurs remarquable que la plupart des motifs agissants que le Moyen Âge emprunte à l'Antiquité, comme les lions et les rinceaux habités, avaient à l'origine une connotation funéraire.

Dans nos exemples, la relation avec l'astrologie, composante importante de la magie médiévale, semble en revanche ténue, voire absente. Nicolas Weill-Parot émet prudemment l'hypothèse selon laquelle Virgile aurait fabriqué son talisman à forme de mouche en tenant compte de l'astrologie: Gervais de Tilbury dit en effet qu'il la façonna arte mathematica ${ }^{41}$. De son côté, Guido Bonatti, astrologue et auteur du traité Liber introductorius ad judicia stellarum, fabriqua plusieurs talismans astrologiques ${ }^{42}$ : cependant, la Chronique de Giovanni de Pedrino ne nous dit pas si les astres

39. Des exemples sont cités par F. Cervini, «Talismani», passim. Sur la perception de l'Antiquité au Moyen Âge et à l'époque moderne, voir Memoria dell'antico nell'arte italiana, dir. Salvatore Settis, 3 vol., Turin, 1984-1986.

40. Le Roman d'Abladane, chap. 28, p. 79.

41. N. Weill-Parot, Les «images astrologiques», p. 149. L'auteur remarque pourtant que l'expression reste ambigüe.

42. Ibid., p. 391-394. 
interviennent dans l'enchantement du bucrane protecteur de la ville. Certes, on doit convenir que le Lion, tout comme le Taureau, sont des signes zodiacaux. Or, si les talismans astrologiques reproduisent souvent la forme «naturelle» des animaux correspondant aux constellations éponymes ${ }^{43}$, dans nos exemples, aucune allusion explicite ne permet d'affirmer que les statues de lions fonctionnent effectivement par l'influx des astres.

Enfin, quand il existe, le renvoi à Dieu et aux saints joue un rôle de premier plan dans l'efficacité de ces images. Les lions «christianisés» de Jaca ou de San Lorenzo in Lucina, figures du Christ, tirent évidemment leur pouvoir de la relation à la divinité. Leur action s'explique alors à l'intérieur d'une logique pleinement chrétienne.

\section{IMAGES AGISSANTES, MAGIE ET «DISPOSITIFS RITUALISANTS »}

Le corpus au cœur de notre étude est très limité et mériterait d'être étoffé. Pour autant, ces quelques exemples semblent autoriser une première réflexion sur les images agissantes aux marges de l'église. Dans le cadre de ce colloque, il est intéressant de se demander en quoi leur fonctionnement peut être défini comme «magique» et en quoi elles peuvent être considérées comme des «objets rituels».

La complexité de la question vient de la variété des définitions que la «magie» et les «rites» ont reçues des anthropologues, des sociologues et des historiens ${ }^{44}$. Les objets au centre des incantations de Virgile, Guido Bonatti ou Flocars semblent rentrer sans difficulté dans la définition d'objets magiques. Mais nous avons vu que d'autres images, pour lesquelles on ne peut supposer l'intervention d'un magicien, ont le même pouvoir de mobiliser et d'orienter des forces occultes, à des fins particulières: cela correspond à la

43. N. Weill-Parot, Les «images astrologiques», passim.

44. Sur ces concepts et la manière dont ils sont appréhendés par les différents courants ethnologiques, sociologiques et psychanalytiques, voir René Alleau, Roger Bastide, «Magie», Encyclopadia Universalis [en ligne: http://www. universalis-edu.com/encyclopedie/magie; consulté le 26 janvier 2014]. Le dictionnaire des sciences humaines, sous la direction de Sylvie Mesure et Patrick Savidan, Paris, PUF, 2006, notamment « Magie et sorcellerie», p. 733-735 (Claude Rivière) et «Rites et rituels», 1024-1026 (Id.). Pour la magie au Moyen Âge, voir encore J.-P. Boudet, Entre science et nigromance. 
définition la plus générale de la «magie». Nous avons montré que ces sculptures peuvent d'ailleurs fonctionner selon certains principes d'efficacité présents dans les traités de magie médiévale, tel celui de la similitudo ou, parfois, de la relation au monde des morts. De ces points de vue, les buts et les fonctionnements des images considérées pourront donc bien être qualifiés de «magiques», dès lors qu'on s'accommode de l'absence du magicien ou de toute fabrication rituelle.

Ces images ne sont pas toutes au centre d'un rite voué à leur infuser un pouvoir, ce qui en ferait inévitablement des «objets rituels». Il n'en reste pas moins que, par leur emplacement, elles marquent un espace et agissent sur les hommes. Capables de rapprocher le franchissement d'un seuil d'un véritable rite de passage ${ }^{45}$, on pourra alors affirmer qu'elles constituent des «dispositifs ritualisants», dans lesquels des hommes, inconsciemment ou consciemment, sont soumis à l'action de forces qui échappent à leur contrôle et au cours normal de la nature. S'ils ne semblent pas répondre à une codification générale, ces «dispositifs ritualisants» manifestent tous leur pouvoir chaque fois que des hommes pénètrent dans leurs espaces d'action.

Mais comment ces images, qui ne relèvent pas forcément d'une thématique chrétienne, qui aspirent à maîtriser les forces sauvages de la nature, qui s'inspirent parfois de motifs antiques, qui sont à même de mettre en scène les défunts et d'être manipulées par des magiciens, étaient-elles perçues par les clercs? Force est de constater qu'elles semblent avoir été globalement acceptées par l'Église. Tout d'abord, même s'il est toujours délicat de tirer parti d'un silence des sources, on peut prudemment avancer que la pénurie de textes sur les pouvoirs des images monumentales, rares par rapport au nombre d'occurrences réelles, semble indiquer que ces pratiques ne faisaient l'objet d'aucune condamnation. De plus, les auteurs qui relatent l'existence de ces images (et en confirment parfois l'efficacité) sont bien souvent des clercs: Conrad de Querfurt, qui décrit la mouche de Virgile, est évêque; Gervais de Tilbury eut également une formation cléricale. Aucune contradiction ne semble exister d'ailleurs dans le fait d'être clerc et magicien: dans le Roman d'Abladane, Flocars est «bon clerc et

45. À ce sujet, voir A. van Gennep, Les rites de passage, p. 19-33. 
maistre d'ingremance $\gg^{46}$; Guido Bonatti œuvre à partir du clocher de l'église abbatiale de Saint-Mercurial, en Toscane, qui lui sert d'observatoire du ciel étoilé: le lieu de son activité est donc l'enclos claustral. En somme, tout laisse croire que le pouvoir de ces images était tacitement accepté et partagé.

Les raisons de cette relative acceptation peuvent être multiples. Par exemple, les sculptures non manipulées par des magiciens s'adressent à des forces déjà présentes dans la nature. L'absence de rituels ou d'invocation aux démons ou à des intelligences supérieures (ce que les spécialistes de la magie appellent «destinativité») fait rentrer le pouvoir de ces images dans une causalité «scientifique»: c'est le même principe que celui de la magie dite «naturelle», théorisée et distinguée de la magie «noire» à partir du XIII ${ }^{\mathrm{e}}$ siècle, et qui ne sera pas perçue comme entrant en contradiction avec le christianisme.

Les seuls cas où ces pratiques semblent avoir ponctuellement posé problème sont ceux où des magiciens interviennent. On sait ainsi que Guido Bonatti a eu un rapport controversé avec le clergé de son époque. S'il jouissait d'une grande renommée, il semble avoir été obligé de débattre publiquement avec le franciscain Hugues de Regio, ce que semble confirmer la méfiance dont l'astrologue fait preuve, dans ses écrits, envers les Frères Mineurs ${ }^{47}$.

En définitive, on pourra donc constater que ces «dispositifs ritualisants» ne répondent à aucun savoir organisé, relèvent de l'occasionnel et acquièrent des formes variables. Cette liberté et cette flexibilité leur permettent probablement de s'intégrer sans heurts aux pratiques de l'Église, de la même manière que ces images agissantes prennent place naturellement dans le décor des édifices, à côté d'autres sujets explicitement chrétiens*.

alessia.trivellone@univ-montp3.fr

Université Paul Valéry - Montpellier 3

UFR 3

Route de Mende

34199 Montpellier cedex 5

46. Le Roman d'Abladane, chap. 21, p. 77.

47. Cf. supra, note 7.

* Pendant la rédaction de cet article, j'ai pu bénéficier d'éclairants échanges avec Laurent Guitton, que je tiens à remercier ici. 


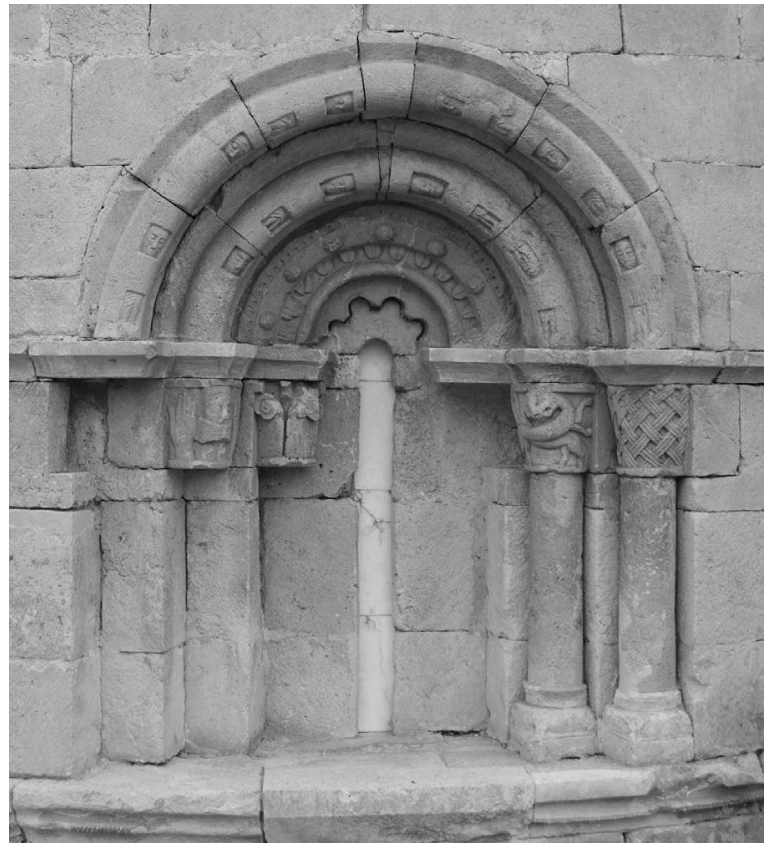

Fig. 1. Église de San Pantaleón de Losa (Burgos), fenêtre (cliché José María Gallardo).

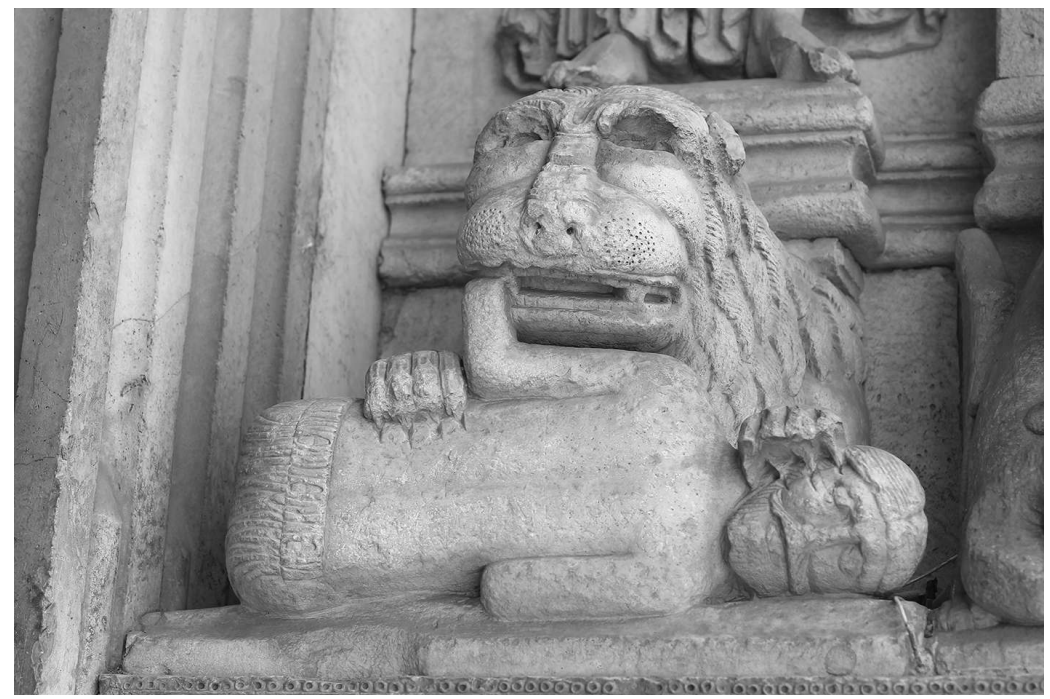

Fig. 2. Arles, église Saint-Trophime, façade. Lion à droite du portail central (cliché Ponyterr). 


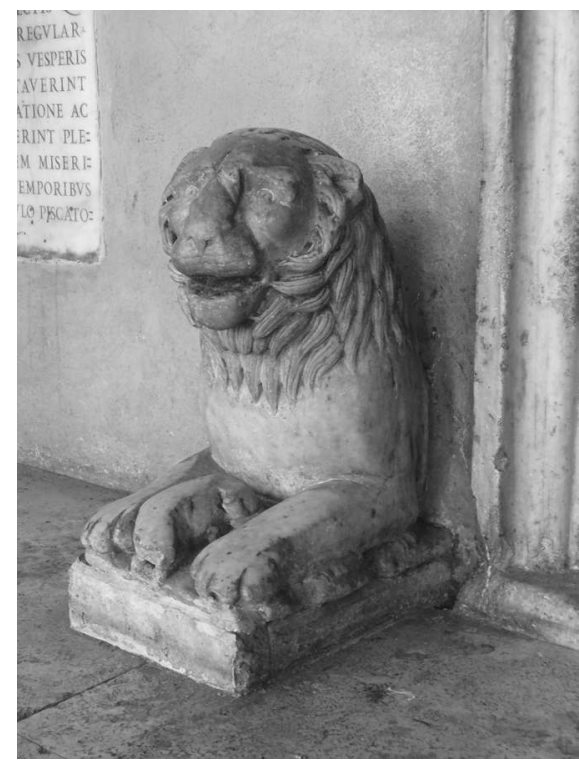

Fig. 3. Rome, Basilique San Lorenzo in Lucina, sculpture à gauche du portail (cliché A. Trivellone).

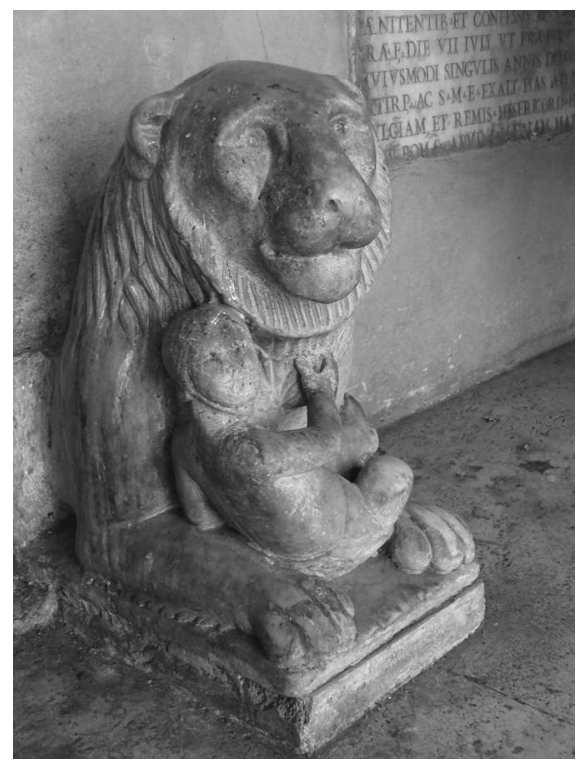

Fig. 4. Rome, Basilique San Lorenzo in Lucina, sculpture à droite du portail (cliché A. Trivellone). 


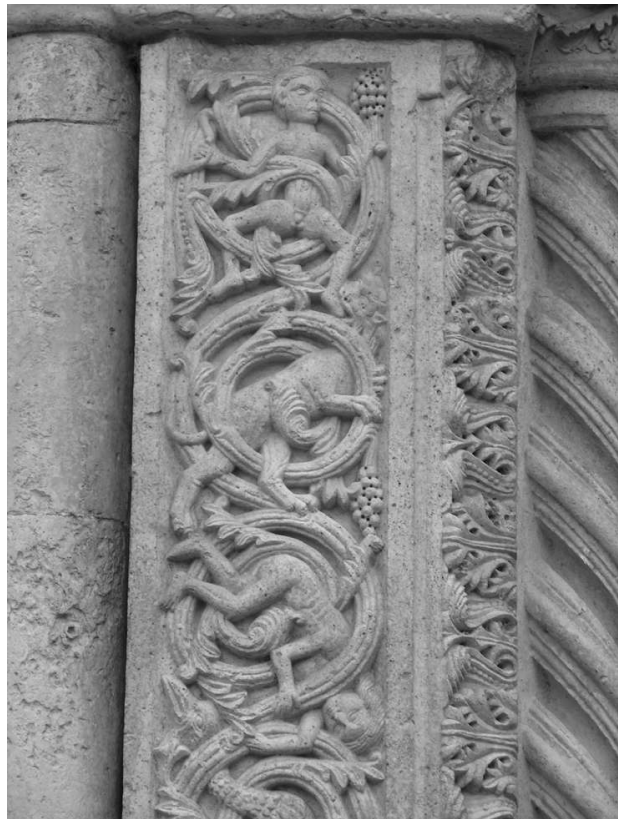

Fig. 5. Trasacco, église Santi Rufino e Cesidio, piédroit gauche du portail dit «des hommes» (détail) (cliché A. Trivellone).

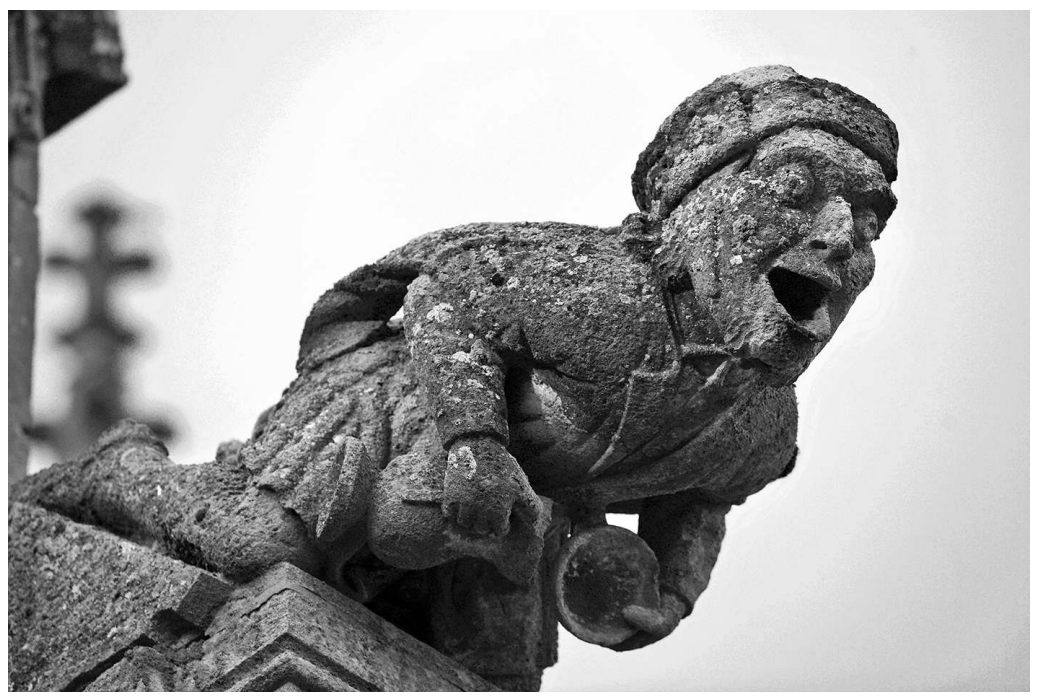

Fig. 6. Basilique Notre-Dame de l'Épine en Champagne, gargouille du chevet (datée de 1515-1524) (cliché Julien Thomas). 